\title{
Perfil dos endodontistas de uma metrópole brasileira quanto ao atendimento odontológico a pacientes com necessidades especiais
}

Gabriela Mancia Gutierrez*; Ana Lurdes Conte Acunha Gonçalves**; Carlos Felipe Bonacina**; Michele Baffi Diniz***; Maria Teresa Botti Rodrigues Santos***; Ângela Toshie Araki Yamamoto***; Adriana de Oliveira Lira***

* Doutora em Odontologia, Programa de Pós-Graduação em Odontologia, Universidade Cruzeiro do Sul

** Doutorando(a) em Odontologia, Programa de PósGraduação em Odontologia, Universidade Cruzeiro do Sul

*** Professora, Departamento de Odontologia, Universidade Cruzeiro do Sul

Recebido: 14/07/2020. Aprovado: 18/11/2020.

\section{RESUMO}

O objetivo do estudo foi caracterizar o perfil dos endodontistas do estado de São Paulo, Brasil em relação ao tratamento odontológico de pacientes com necessidades especiais (PNE). Um questionário online sobre capacitação do profissional e dados sobre o atendimento odontológico de PNE foi enviado para 3.500 endodontistas registrados no Conselho Regional de Odontologia de São Paulo. Dentre os 138 profissionais que retornaram, $57,2 \%$ eram do sexo feminino; 34,8\% estavam na faixa etária entre 31 e 40 anos; 42,8\% tinham entre 11 e 20 anos de formados; e 38,4\% possuíam 11 a 20 anos de especialidade. Apenas 22,5\% eram capacitados para o atendimento de PNE, sendo que desses, 58,1\% tiveram apenas aula teórica. Dos que não receberam capacitação na pós-graduação, 80,4\% gostariam de ter recebido. Com relação ao interesse em cursos de atendimento a PNE após a especialização, observou-se que apenas 15,9\% realizaram algum tipo de curso, a maioria apenas teórico. Em relação às dificuldades durante o atendimento de PNE a "falta de colaboração do paciente durante o atendimento" foi citada por 74,6\%, a "insegurança devido à falta de preparo profissional" por 55,0\%. A análise da associação das variáveis pelo teste Qui-quadrado apontou que quanto maior o tempo de formação, menor essa dificuldade $(\mathrm{p}=0,0415)$. Os especialistas encontram mais dificuldades comparados aos que tem formação associada (mestrado e doutorado) ( $\mathrm{p}=0,0369)$. Não houve associação significativa entre as dificuldades e o tipo de instituição de formação (pública ou privada) ( $\mathrm{p}>0,05$ ). O panorama atual da formação do endodontista não contempla o atendimento as pessoas com necessidades especiais.

Descritores: Educação de Pós-Graduação em Odontologia. Assistência Odontológica para Pessoas com Deficiências. Acesso aos Serviços de Saúde. Endodontia 


\section{INTRODUÇÃO}

A Organização Mundial da Saúde (OMS) estima que $15 \%$ da população mundial vive com alguma deficiência ${ }^{1}$. No Brasil, segundo o Instituto Brasileiro de Geografia e Estatística (IBGE) no ano de $2010^{2}, 45,6$ milhões de pessoas $(23,9 \%)$ possuem algum tipo de deficiência ou incapacidade. Os pacientes com necessidades especiais (PNE) ou com deficiência apresentam uma ou mais limitações, temporárias ou permanentes, de ordem mental, física, sensorial, emocional, de crescimento ou médica, que os impede de ser submetidas a intervenções odontológicas convencionais ${ }^{3}$.

A Odontologia para pacientes com necessidades especiais (OPNE) envolve o gerenciamento de cuidados da saúde bucal em pessoas com deficiência intelectual, sistêmicas, físicas ou psiquiátricas e tornou-se uma especialidade no Brasil em 2002 ${ }^{4}$ O tratamento odontológico para o PNE exige planejamento baseado nas deficiências e particularidades do paciente, fazendo-se necessário de manejo comportamental, conhecimento científico e modificações das técnicas para o sucesso do tratamento $^{5,6}$.

Há evidências de variação nacional e internacional na disponibilidade de educação e treinamento em nível de graduação na área de OPNE, sendo que a disciplina ainda não é de caráter obrigatório nas instituições ${ }^{7}$. Nos Estados Unidos e Canadá, 65 escolas de Odontologia foram questionadas quanto à educação dos alunos de graduação sobre a temática de OPNE e 22 instituições responderam que abordam a temática de OPNE durante a graduação, sendo que $64 \%$ responderam que ocorre de forma obrigatória ${ }^{5}$. No Brasil, das 221 instituições de ensino superior com curso de Odontologia, $56(27,7 \%)$ oferecem a referida disciplina, sendo que 34 delas $(60,7 \%)$ oferecem a disciplina na modalidade obrigatória, 14 (25\%) na modalidade optativa e em 8 instituições a mesma não está disponível ${ }^{8}$.

Os cirurgiões-dentistas $(\mathrm{CD})$ provavelmente se depararão com um número significativo de PNE, no decorrer da sua vida profissional ${ }^{7}$, uma vez que este grupo de indivíduos apresenta maior risco para o acometimento de doenças bucais. Tal panorama ocorre em função do uso sistêmico de medicamentos, hábitos alimentares precários ${ }^{9}$, dificuldade na realização do controle de placa bacteriana $^{10}$, traumatismos dentários causados por quedas e associados ao aumento do overjet e falta de selamento labial ${ }^{11}$. Entre os diversos tipos de cuidados odontológicos, o tratamento endodôntico é uma das necessidade do PNE, devido a lesões cariosas e traumatismo dentário.

Diante destas considerações, o presente estudo teve como objetivo caracterizar o perfil dos CD especialistas em endodontia do estado de São Paulo em relação ao tratamento odontológico de PNE.

\section{MATERIAIS E MÉTODOS}

Foram incluídos no estudo todos os CD especialistas em endodontia com inscrição ativa no Conselho Regional de Odontologia de São Paulo (CROSP), que possuíssem qualquer tempo de formado e que aceitassem participar voluntariamente da pesquisa, concordando com o Termo de Consentimento Livre e Esclarecido e respondendo ao questionário online.

O estudo foi do tipo exploratório descritivo, com delineamento transversal e abordagem quantitativa. Inicialmente, foi enviado um ofício ao Presidente do CROSP, explicando os objetivos da pesquisa e solicitando o envio dos questionários online aos $\mathrm{CD}$ especialistas em endodontia com inscrição ativa no estado. 
O instrumento de coleta de dados consistiu em questionário estruturado, autoaplicável, de fácil compreensão, criado pelos próprios pesquisadores na plataforma Google forms, contendo 15 questões de múltipla escolha ou dicotômicas do tipo "SIM" ou "NÃO". O acesso ao questionário ocorreu por meio de um link enviado para o e-mail cadastrado dos endodontistas pelo CROSP.

A pesquisa foi aprovada pelo Comitê de Ética institucional, CAAE $\mathrm{n}^{\text {o }}$ 67128517.9.0000.5373.

Os dados foram processados utilizando o programa SPSS v. 23.0 (IBM, Amronk, NY, EUA). Os dados descritivos foram analisados por distribuição de frequências absoluta (n) e relativa (\%). O teste do Qui-quadrado foi utilizado com nível de significância de $5 \%(\mathrm{p}<0,05)$.

\section{RESULTADOS}

A amostra constituiu-se dos endodontistas que responderam ao questionário. Dos 3500 questionários enviados, 138 foram válidos, representando uma taxa de resposta de 3,94\%.

Quanto à caracterização do perfil profissional dos CD especialistas em endodontia do estado de São Paulo respondentes (tabela 1), observou-se que a amostra foi predominantemente feminina $(57,2 \%)$; a faixa etária mais prevalente foi de 31 a 40 anos (34,8\%); a maioria graduou-se em instituições privadas (61,3\%), com 11 a 20 anos de formado (42,8\%), sendo que $53 \mathrm{CD}$ $(38,4 \%)$ tinham o título de especialista em endodontia há 11 - 20 anos. A maioria dos profissionais, $88(63,8 \%)$ exerciam suas atividades no serviço privado e $86(62,3 \%)$ atuavam como generalistas e especialistas em endodontia.
Na tabela 2 pode-se observar os dados sobre a capacitação profissional dos endodontistas. A maior parte dos participantes da pesquisa $(65,9 \%)$ possuía exclusivamente o curso de especialização em endodontia, sendo que apenas um participante $(0,7 \%)$ também possuía especialização em Odontologia para Pacientes com Necessidades Especiais (OPNE). A respeito da capacitação dos endodontistas em OPNE durante a pós-graduação, somente $31(22,5 \%)$ afirmaram ter recebido algum tipo de treinamento, e destes, $18(58,1 \%)$ relataram ter recebido apenas aulas teóricas. Dos que não receberam capacitação em OPNE na pósgraduação, quando questionados sobre o interesse em tê-la recebido no respectivo período, a maioria dos CD (80,4\%), respondeu afirmativamente. Com relação ao interesse em cursos de OPNE após a especialização em endodontia, observou-se que apenas $22(15,9 \%)$ realizaram algum tipo de curso, sendo que a maioria destes realizaram cursos apenas cursos teóricos $(52,4 \%)$.

Quando questionados sobre as maiores dificuldades durante o atendimento odontológico de PNE, os participantes podiam assinalar mais de uma resposta (tabela 3), sendo dadas 6 opções de respostas. A "falta de colaboração do paciente durante o atendimento" foi citada como a maior dificuldade (50\%), seguida de "falta de recursos técnicos e humanos que viabilizem o atendimento" $(33,3 \%)$ e "insegurança devido à falta de preparo profissional" (30,4\%). Quando questionados para onde os profissionais encaminhavam os PNE quando não conseguiam atender os mesmo, 39,7\% afirmaram encaminhá-los para serviços especializados e para colegas que os atendam. Apenas 5,3\% relataram não encaminhar, pois não sabem para onde direcionar estes pacientes. 
Tabela 1. Caracterização da amostra

\begin{tabular}{|c|c|c|}
\hline Variáveis & $\mathbf{n}$ & $\%$ \\
\hline \multicolumn{3}{|l|}{ Sexo } \\
\hline Masculino & 59 & 42,8 \\
\hline Feminino & 79 & 57,2 \\
\hline \multicolumn{3}{|l|}{ Faixa etária } \\
\hline $20-30$ & 20 & 14,5 \\
\hline $31-40$ & 48 & 34,8 \\
\hline $41-50$ & 39 & 28,3 \\
\hline $51-60$ & 25 & 18,1 \\
\hline$>$ de 60 & 6 & 4,3 \\
\hline \multicolumn{3}{|c|}{ Instituição de Ensino Superior de graduação } \\
\hline Privada & 84 & 61,3 \\
\hline Pública & 53 & 38,7 \\
\hline \multicolumn{3}{|c|}{ Tempo de formado (anos) } \\
\hline$<5$ & 11 & 8,0 \\
\hline 5 a 10 & 14 & 10,1 \\
\hline 11 a 20 & 59 & 42,8 \\
\hline$>20$ & 54 & 39,1 \\
\hline \multicolumn{3}{|c|}{ Tempo desde a obtenção do título de endodontista } \\
\hline$<5$ & 26 & 18,8 \\
\hline 5 a 10 & 30 & 21,7 \\
\hline 11 a 20 & 53 & 38,4 \\
\hline$>20$ & 29 & 21,0 \\
\hline \multicolumn{3}{|c|}{ Atuação no exercício profissional } \\
\hline Generalista & 4 & 2,9 \\
\hline Somente especialista & 48 & 34,8 \\
\hline Ambos & 86 & 62,3 \\
\hline \multicolumn{3}{|c|}{ Serviço de atuação profissional } \\
\hline Público & 9 & 6,5 \\
\hline Privado & 88 & 63,8 \\
\hline Ambos & 41 & 29,7 \\
\hline
\end{tabular}


Tabela 2. Capacitação profissional dos endodontistas

\begin{tabular}{lcc}
\hline Variáveis & n & \% \\
\hline Pós-graduação & & \\
$\quad$ Apenas especialização & 91 & 65,9 \\
Especialização e Mestrado & 29 & 21,0 \\
Especialização, Mestrado e Doutorado & 18 & 13,0 \\
\hline Recebeu treinamento, na pós-graduação, em OPNE? & & \\
Sim & 31 & 22,5 \\
Não & 107 & 77,5 \\
\hline De que forma esse treinamento, em OPNE, ocorreu? & & \\
Aula teórica & 18 & 58,1 \\
Aula prática & 2 & 6,5 \\
Teórica e prática & 11 & 35,5 \\
\hline Caso não tenha recebido o treinamento, gostaria de ter recebido? & & \\
Sim & 86 & 80,4 \\
Não & 21 & 19,6 \\
\hline Realizou curso de OPNE, após a especialização? & & \\
Sim & 22 & 15,9 \\
Não & 116 & 84,1 \\
\hline Se “SIM” de que forma? & & \\
Teórico & 11 & 52,4 \\
Atualização & 9 & 42,9 \\
Especialização & 1 & 4,8 \\
\hline
\end{tabular}

Tabela 3. Atendimento odontológico de PNE pelos endodontistas

\begin{tabular}{lcc}
\hline Variáveis & n & \% \\
\hline Quais as dificuldades no atendimento de PNEs? & & \\
Falta de colaboração do paciente durante o atendimento & 69 & 50,0 \\
Falta de recursos técnicos e humanos que viabilizem o atendimento & 46 & 33,3 \\
Insegurança devido à falta de preparo profissional & 42 & 30,4 \\
Remuneração não compatível com o trabalho & 24 & 17,4 \\
Todas as dificuldades citadas acima & 34 & 24,6 \\
Outras respostas & 16 & 11,6 \\
\hline Para onde encaminha quando não consegue atender? & & \\
Para serviços especializados e para colegas que os atendam & 52 & 39,7 \\
Para serviços especializados & 45 & 34,4 \\
Para colegas que os atendam & 27 & 20,6 \\
Não encaminha, pois não sabe para onde encaminhá-los & 7 & 5,3 \\
\hline
\end{tabular}


Por meio da análise da associação das variáveis pelo teste Qui-quadrado (tabela 4) observou-se que quanto maior o tempo de formação, devido à menor a dificuldade no pela "insegurança devido à falta de preparo profissional" $\quad(\mathrm{p}=0,0415) . \quad$ Os especialistas assinalaram mais a opção 5 "Todas as dificuldades citadas acima", comparados aos que tem formação associada (mestrado e doutorado) $(\mathrm{p}=0,0369)$. Não houve associação significativa entre as dificuldades e o tipo de instituição de ensino superior IES (pública ou privada) ( $p>0,05)$.

Tabela 4. Associação entre tempo de formação, formação acadêmica do profissional; e dificuldade no atendimento a pacientes com deficiência

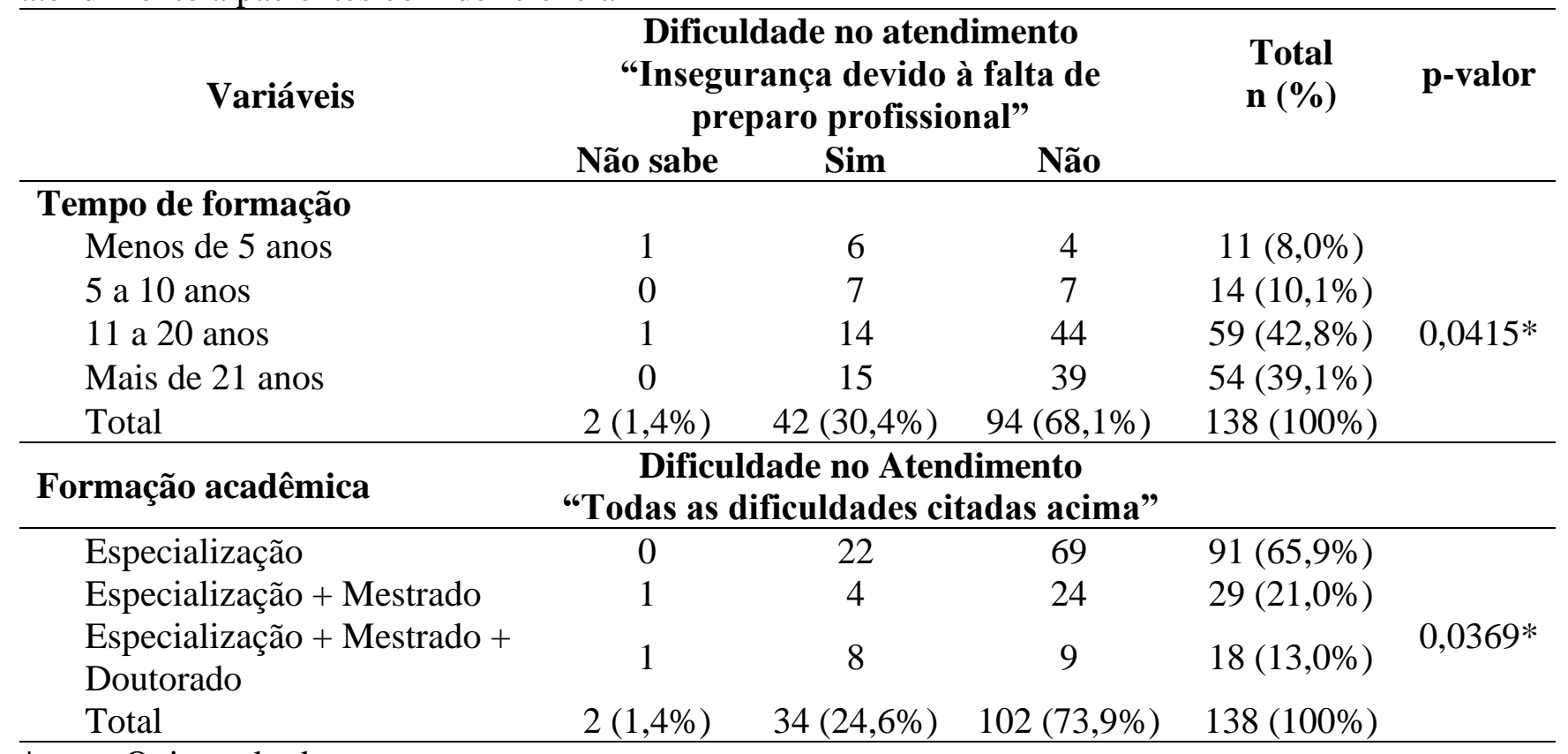

* teste Qui-quadrado

\section{DISCUSSÃO}

O estado de São Paulo é o mais populoso do Brasil, tendo a maior concentração de CD do país $(29,4 \%)^{12}$. De todos os endodontistas do país, uma grande parte se encontra neste estado $(24,2 \%)$, sendo estes na grande maioria $(65,3 \%)$ do sexo feminino assim como se observou na presente amostra. Dos 29.189 CDs especialistas em diversas áreas da Odontologia do estado de São Paulo, apenas $256(0,9 \%)$ são especialistas em $\mathrm{OPNE}^{13}$, fazendo-se importante os profissionais de outras especialidades se capacitarem no atendimento a PNE para atender a demanda do mercado, uma vez que o número de pessoas com deficiências tende a aumentar a cada ano.

Além disso, com o aumento da expectativa de vida dos PNE e a necessidade e o interesse em manter mais os dentes na boca, os CD devem estar dispostos, mais bem equipados e preparados para fornecer o tratamento endodôntico a esses pacientes ${ }^{14}$, assim como os endodontistas devem 
utilizar técnicas de sedação consciente, anestesia geral e optar por tratamento endodôntico em sessão única, quando viável.

Um estudo na Austrália verificou que todos os CD especialistas em OPNE e 95,7\% dos endodontistas afirmaram que já haviam realizado tratamento endodôntico em PNE, enquanto pouco mais da metade dos CD clínicos gerais $(51,4 \%)$ já o haviam realizado. Dentre as razões dadas pelos profissionais por não realizarem tratamento endodôntico em PNE, a cooperação limitada foi relatada por $64,4 \%$ e os movimentos involuntários por $51,5 \%^{14}$. O presente estudo também evidenciou que a maior dificuldade encontrada pelos endodontistas foi do próprio comportamento do PNE, dificultando a colaboração durante o atendimento.

Foi observado ainda que "insegurança devido à falta de preparo profissional" foi uma das dificuldades relatadas pelos endodontistas. A maioria dos cuidadores de PNE considera a falta de capacitação do $\mathrm{CD}$ como a maior barreira de acesso ao tratamento odontológico ${ }^{15,16}$. Sendo assim, torna-se evidente a necessidade de capacitação profissional, não só de forma teórica, mas também clínica, visando melhorar a qualidade no atendimento e possibilidade de tratamento endodôntico dos PNE. Nesta amostra os profissionais que eram somente especialistas em endodontia relatavam mais dificuldades no atendimento a PNE quando comparados aos que tem formação associada (mestrado e doutorado) $(\mathrm{p}=0,0369)$, ficando clara a importância da continuidade da aprendizagem e capacitação profissional.

O sistema de ensino superior no Brasil é dividido em instituições privadas e públicas. As universidades públicas são financiadas pelo governo, não tendo nenhum custo financeiro para o estudante, sendo o processo de seleção geralmente mais competitivo em relação ao das instituições privadas. Professores e pesquisadores em universidades públicas brasileiras são concursados e possuem salários mais altos em comparação àqueles das instituições privadas. Segundo o Ministério de Educação, existiam em São Paulo, quando da submissão deste artigo, 56 instituições com oferta do curso de graduação em Odontologia, sendo 9 públicas e 45 privadas $^{17}$. Em 2018 as três melhores faculdades de odontologia do estado de São Paulo foram públicas ${ }^{18}$. A formação em uma instituição pública ou privada não esteve associada à dificuldade durante o atendimento do PNE pelos respondentes ( $p>0,05)$.

É de grande importância a exposição precoce à experiência clínica envolvendo PNE e introdução da disciplina de OPNE no currículo da graduação $^{6}$. O número de universidades que incluem a disciplina regular de OPNE nos cursos de graduação é muito inferior à necessidade do $\mathrm{Brasil}^{8}$. Esta deficiência pode ser pior nos cursos de pós-graduação, pois apenas $22,5 \%$ da presente amostra afirmou ter recebido algum tipo de treinamento em OPNE, número muito baixo para a grande necessidade odontológica que estes pacientes apresentam.

Os PNE têm o direito de receber tratamento odontológico de maneira semelhante à população em geral. Uma abordagem multidisciplinar no manejo dos PNE que necessitem de tratamento endodôntico fornece a este grupo de pacientes a oportunidade de manter os dentes ${ }^{14}$, deixando procedimentos como exodontia como o último recurso de tratamento odontológico.

\section{CONCLUSÃO}

O panorama atual da formação do endodontista no estado de São Paulo, Brasil, não 
contempla $\mathrm{o}$ atendimento às pessoas com necessidades especiais. A introdução da temática da OPNE ainda na graduação faz-se necessária para despertar o interesse em conhecimento científico teórico e prático nos futuros profissionais da Odontologia, generalistas e especialistas.

\section{ABSTRACT \\ Profile of endodontists in a Brazilian metropolis regarding dental care for patients with special needs}

The aim of this study was to characterize the profile of endodontic professionals from the state of São Paulo, Brazil in relation to dental treatment for patients with special needs (PSN). An online questionnaire on professional training and data on PSN dental care was sent to 3,500 endodontic professionals registered with the São Paulo Regional Dental Council in 2017. Of the 138 returning professionals, $57.2 \%$ were female; $34.8 \%$ aged 31 40 years; $42.8 \%$ had 11-20 years since graduation; $38.4 \%$ had $11-20$ years of endodontic specialization. Only $22.5 \%$ were trained to provide dental care to PSNs and of these, $58.1 \%$ had only theoretical classes. Of those who did not receive postgraduation training, $80.4 \%$ would like to have received it. Regarding the interest in PSN care courses after specialization, it was observed that only $15.9 \%$ performed some type of course, most of them theoretical only. Regarding difficulties during PSN care, "lack of patient collaboration during care" was mentioned by $74.6 \%$, and "insecurity due to lack of professional preparation" by $55.0 \%$. The analysis of the association of variables by the Chi-square test showed that the longer the training time, the lower the difficulty during PSN care $(\mathrm{p}=0.0415)$. Specialists find more difficulties compared to those with associated training (masters and doctorate) $(\mathrm{p}=0.0369)$. There was no significant association between difficulties in PSN care and type of HEI (public or private) $(\mathrm{p}>0.05)$. The current panorama of endodontic professional training does not seem to include care for patients with special needs.

Descriptors: Education, Dental, Graduate. Dental Care for Disabled. Health Services Accessibility. Endodontics.

\section{REFERÊNCIAS}

1. World Health Organization. World report on disability. 2012. [Acesso em: 02/06/2019]. Disponível em: http://www.pessoacom deficiencia.sp.gov.br/usr/share/documents/R ELATORIO_MUNDIAL_COMPLETO.pdf.

2. Instituto Brasileiro de Geografia e Estatística. Pessoas com deficiência no estado de São Paulo censo demográfico - 2010. Rio de Janeiro: Instituto Brasileiro de Geografia e Estatística; 2010. [Acesso em: 02/06/2019]. Disponível em: http://www.pessoacomdefi ciencia.sp.gov.br/Content/uploads/20131213 114958 2013analise_censo_EstSP.pdf.

3. Ministério da Saúde. Saúde Bucal. Caderno de Atenção Básica, n 17. 2008. [Acesso em: 02/06/2019]. Disponível em: http://bvsms. saude.gov.br/bvs/publicacoes/saude_bucal.pdf.

4. Conselho Federal de Odontologia. Resolução CFO-25/2002, 16 de maio de 2002. Estabelece as áreas de competência para atuação dos especialistas em Disfunção Têmporo-Mandibular e Dor Orofacial; Odontogeriatria; Odontologia do Trabalho; Odontologia para Pacientes com Necessidades Especiais e em Ortopedia Funcional dos Maxilares e dá outras providências. Diário Oficial da União 2002; maio 28.

5. Krause M, Vainio L, Zwetchkenbaum S, Inglehart MR. Dental Education About Patients with Special Needs: A Survey of U.S. 
and Canadian Dental Schools. J Dent Educ. 2010;74(11):1179-89.

6. Ahmad MS, Razak IA, Borromeo GL. Special Needs Dentistry: perception, attitudes and educational experience of Malaysian dental students. Eur J Dental Educ. 2015;19:44-52.

7. Dougall A, Pani SC, Thompson S, Faulks D, Romer M, Nunn J. Developing an undergraduate curriculum in Special Care Dentistry - by consensus. Eur J Dent Educ. 2017;7:46-56.

8. Bonato LL, Lopes AMS, Silva CM, Itner RG, Silva ACH. Situação atual da formação para assistência de pessoas com necessidades especiais nas faculdades de odontologia no Brasil. ClipeOdonto. 2013;5(1):10-5.

9. Thikkurissy S, Lal S. Oral Health Burden in Children with Systemic Diseases. Dent Clin North Am. 2009;53(2):351-7.

10. Sinha N, Singh B, Chhabra KG, Patil S. Comparison of oral health status between children with cerebral palsy and normal children in India: A case-control study. J Indian Soc Periodontol. 2015;19(1):78-82.

11. Al-Batayneh OB, Owais AI, Al-Saydali MO, Waldman HB. Traumatic dental injuries in children with special health care needs. Dental Traumatol. 2017;33(4):269-75.

12. Conselho Federal de Odontologia. Quantidade geral de profissionais e entidades ativas. 2019. [Acesso em: 02/06/2019]. Disponível em: http://cfo.org.br/website lestatisticas/quantidade-geral-de-entidades-eprofissionais-ativos/.
13. Conselho Regional de Odontologia de São Paulo. Especialidades. [Acesso em: 02/06/2019]. Disponível em: http://www.crosp.org.br/intranet/estatisticas/e stEspecialistas.php.

14. Yap E, Parashos P, Borromeo GL. Root canal treatment and special needs patients. Int Endod J. 2014;48(4):351-61.

15. Leal Rocha LL, Saintrain MVL, Vieira-Meyer APGF. Access to dental public services by disabled Persons. BMC Oral Health. 2015;15:35.

16. Williams JJ, Spangler CC, Yusaf NK. Barriers to dental care access for patients with special needs in an affluent metropolitan community. Spec Care Dentist. 2015;35(4):190-96.

17. Ministério de Educação e Cultura (MEC). Cadastro Nacional de Cursos e Instituições de Educação Superior Cadastro e-MEC. 2019. [Acesso em: 02/06/2019]. Disponível em: http://emec.mec.gov.br/.

18. Folha de São Paulo. RUF ranking universitário folha. 2018. [Acesso em: 02/06/2019]. Disponível em: https://ruf.folha.uol.com.br/2018/ranking-decursos/odontologia/.

Correspondência para:

Carlos Felipe Bonacina

e-mail: felipebonacina@msn.com

Estrada de Santa Isabel, 1201 - Sala 119

Caputera

07435-180 Arujá/SP 